\title{
Are chest compression depths measured by the Resusci Anne SkillReporter and CPRmeter the same?
}

\author{
CHAN WOONG KIM, JE HYEOK OH \\ Department of Emergency Medicine, College of Medicine, Chung-Ang University, Seoul, Republic of Korea \\ Correspondence to \\ Je Hyeok $\mathrm{OH}$ \\ Department of Emergency Medicine \\ College of Medicine, Chung-Ang University \\ 84 Heukseok-ro, Dongjak-gu, 06974 Seoul, Republic of Korea \\ Phone/Fax: 82-2-6299-1820, 82-2-6299-2558 \\ E-mail:jehyeok.oh@gmail.com; jehyeokoh@cau.ac.kr \\ IRB approval number: Chung-Ang University Hospital Institutional Review Board; C2015024(1482) \\ Trial registration: Clinical Research Information Service; KCT0001420
}

\section{ABSTRACT}

Objective. We investigated whether data collected using the Resusci Anne SkillReporter were comparable with those collected using the CPRmeter (cardiopulmonary resuscitation meter -an accelerometer feedback device used to provide high-quality chest compressions).

Materials and Methods. Fifty continuous chest compressions were performed using a Resusci Anne SkillReporter and a CPRmeter under two conditions (Experiment 1: complete chest wall recoil; Experiment 2: incomplete chest wall recoil). The conditions were defined according to visual feedback signals provided by the CPRmeter. A single healthcare worker performed 20 repetitions under each experimental condition alternately. Chest compression data were collected and analyzed using the Laerdal PC SkillReporting System and QCPR Review software.

Results. The mean difference in chest compression depth between the Resusci Anne SkillReporter and CPRmeter was $6.7 \pm 1.2$ $\mathrm{mm}$ in Experiment 1 (95\% CI: 6.1 7.3) and was significantly higher in Experiment $2(17.3 \pm 1.9 \mathrm{~mm} ; 95 \%$ CI: $16.4 \sim 18.2 ; \mathrm{p}<$ $0.001)$.

Conclusions. The chest compression depth measured by the Resusci Anne SkillReporter was significantly different from that of the CPRmeter. Cardiopulmonary resuscitation instructors, trainees, and researchers should be aware of this difference to ensure the most accurate interpretation of their training or experimental results.

Key words: cardiopulmonary resuscitation, manikins, feedback, education, training

\section{INTRODUCTION}

The 2015 International Consensus on Cardiopulmonary Resuscitation (CPR) and Emergency Cardiovascular Care Science with Treatment Recommendations suggested the use of real-time feedback devices for CPR training because they provide students effective feedback. (1) The Resusci Anne SkillReporter is widely used for CPR training because it enables the objective measurement and reporting of chest compression and ventilation data.

In this study, we investigated whether data collected using the Resusci Anne SkillReporter were comparable with those collected using another measurement device. Recently, we found that chest compression depth measured using the Resusci Anne SkillReporter differed from that of the CPRmeter (an accelerometer feedback device used to provide high-quality chest compressions) by more than $20 \%$ during CPR training sessions. We concluded that this discrepancy was not due to chance, because it exceeded the allowable error range and was observed repeatedly. Furthermore, the complete recoil rates were very low $(2-45 \%)$ in these data.

We hypothesized that chest compression depths measured by the Resusci Anne SkillReporter and the CPRmeter were different and that the differences increased under an incomplete recoil condition. This study was conducted to evaluate our hypothesis and identify the reasons for the discrepancy.

\section{MATERIALS AND METHODS}

\section{Study design}

Our prospective, non-randomized trial was conducted using two conditions. Experiment 1 was conducted under a complete recoil condition, and Experiment 2 was conducted under an incomplete recoil condition. The conditions were defined according to visual feedback signals provided by the CPRmeter (Laerdal Medical, Stavanger, Norway). The chest compressions under each condition were recorded simultaneously using a Resusci Anne SkillReporter (Laerdal Medical) and CPRmeter. The study was approved by the Chung-Ang University Hospital Institutional Review Board (Approval number: C2015024 [1482]) and registered in the World Health Organization International Clinical Trial Registry Platform (Clinical Research Information Service; registered number: KCT0001420).

\section{Study setting and materials}

The experiments were performed on a hard floor without a mattress. Fifty continuous chest compressions without ventilation were performed on the manikin in each experiment. A single healthcare worker performed 20 repetitions under each experimental condition alternately. A 10-min resting period was allowed between experiments. Chest compression data were collected and analyzed using the Laerdal PC SkillReporting System (Laerdal Medical) and QCPR Review software (ver. 3.1; Laerdal Medical).

The sample size was calculated based on chest compression depth as the primary outcome variable. In a previous study, we 
determined that the mean chest compression depth $(\mathrm{MCD}) \pm$ standard deviation was $53.9 \pm 5.8 \mathrm{~mm}$. (2) We set the twosided significance level at 0.05 and the statistical power at $80 \%$. Under the hypothesis that the expected MCD difference between the Resusci Anne SkillReporter and CPRmeter was $10 \%$, the allowable difference was set at $5.39 \mathrm{~mm}$. The minimum number of experiments was determined to be 10 using web-based software (sample size calculator: one sample mean). (3)

\section{Outcome variables}

The primary outcome variable was the mean difference in chest compression depth between the Resusci Anne SkillReporter and CPRmeter. The secondary outcome variables were mean compression rate/min, adequate rate (\%), adequate depth (\%), and complete release (\%).

\section{Statistical analysis}

Chest compression data from the Resusci Anne SkillReporter and the CPRmeter were compared in each experiment. Statistical evaluations were performed using PASW Statistics software ver. 18.0 (SPSS, Inc., Chicago, IL, USA). Data are presented as means with standard deviations. The outcome variables were analyzed using a Shapiro-Wilk test to verify a normal distribution. A two-sided paired t-test was used for data with a normal distribution; otherwise, a Wilcoxon signed-rank test was used. A P-value $<0.05$ was considered to indicate statistical significance.

\section{RESULTS}

\section{Mean differences in chest compression depth}

The mean difference in chest compression depth between the Resusci Anne SkillReporter and CPRmeter was $6.7 \pm 1.2 \mathrm{~mm}$ in Experiment 1 (95\% CI: 6.1 7.3; table 1) and was significantly higher in Experiment $2(17.3 \pm 1.9 \mathrm{~mm} ; 95 \% \mathrm{CI}: 16.4 \sim 18.2 ; \mathrm{p}<$ $0.001)$.

\section{Comparisons of Resusci Anne Skill- Reporter and CPRmeter data}

The Resusci Anne SkillReporter and CPRmeter chest compression data are shown in table 2 .

The mean chest compression depth and percentages of adequate depth and complete release measured using the Resusci

Table 1. The mean difference in chest compression depth between the Resusci Anne SkillReporter and CPRmeter.

\begin{tabular}{llllll}
\hline & $\begin{array}{l}\text { Experiment } \\
\mathbf{1}(\mathbf{n}=\mathbf{2 0})\end{array}$ & $\begin{array}{l}\text { Experiment } \\
\mathbf{2 ( n = 2 0 )}\end{array}$ & $\mathbf{9 5 \%}$ CI of the difference & \\
\hline Parameters & $(95 \%$ CI $)$ & $(95 \%$ CI $)$ & Lower limit & Upper limit & P-value \\
\hline Difference & $6.7 \pm 1.2$ & $17.3 \pm 1.9$ & -11.634 & -9.566 & $<0.001$ \\
& $(6.1$ to 7.3$)$ & $(16.4$ to 18.2$)$ & & & \\
\hline
\end{tabular}

A P-value $<0.05$ is presented in bold.

Table 2. Comparisons of Resusci Anne SkillReporter and CPRmeter data.

\begin{tabular}{|c|c|c|c|}
\hline & \multicolumn{3}{|c|}{ Experiment 1 (Complete chest wall recoil, $n=20)$} \\
\hline & Resusci Anne SkillReporter & CPRmeter & P-value \\
\hline Mean compression depth (mm) & $58.6 \pm 1.1$ & $51.9 \pm 0.9$ & $<0.001$ \\
\hline Mean compression rate (/min) & $111.4 \pm 1.6$ & $111.5 \pm 1.6$ & 0.317 \\
\hline Adequate rate $(\%)$ & $99.7 \pm 1.3$ & $99.8 \pm 1.1$ & 0.317 \\
\hline Adequate depth (\%) & $100.0 \pm 0.0$ & $88.7 \pm 10.4$ & $<0.001$ \\
\hline Complete release (\%) & $100.0 \pm 0.0$ & $99.1 \pm 2.0$ & 0.039 \\
\hline Corrected hand-position (\%) & $100.0 \pm 0.0$ & N/A & $\mathrm{N} / \mathrm{A}$ \\
\hline Good compression (\%) & N/A & $88.3 \pm 10.3$ & $\mathrm{~N} / \mathrm{A}$ \\
\hline \multirow[t]{3}{*}{ Flow time (\%) } & N/A & $98.0 \pm 0.0$ & N/A \\
\hline & \multicolumn{3}{|c|}{ Experiment 2 (Incomplete chest wall recoil, $n=20$ ) } \\
\hline & Resusci Anne SkillReporter & CPRmeter & P-value \\
\hline Mean compression depth (mm) & $55.3 \pm 1.5$ & $38.0 \pm 2.1$ & $<0.001$ \\
\hline Mean compression rate (/min) & $113.2 \pm 1.1$ & $113.4 \pm 0.9$ & 0.279 \\
\hline Adequate rate (\%) & $99.4 \pm 1.5$ & $99.4 \pm 1.8$ & 0.705 \\
\hline Adequate depth (\%) & $99.7 \pm 1.0$ & $0.2 \pm 0.6$ & $<0.001$ \\
\hline Complete release (\%) & $6.8 \pm 5.4$ & $1.9 \pm 0.9$ & $<0.001$ \\
\hline Corrected hand-position (\%) & $100.0 \pm 0.0$ & N/A & $\mathrm{N} / \mathrm{A}$ \\
\hline Good compression (\%) & N/A & $0.0 \pm 0.0$ & $\mathrm{~N} / \mathrm{A}$ \\
\hline Flow time (\%) & N/A & $98.0 \pm 0.0$ & N/A \\
\hline
\end{tabular}

N/A: not applicable, A P-value $<0.05$ is presented in bold.

Anne SkillReporter were significantly higher than those measured using the CPRmeter in both experiments (table 2).

\section{Comparison of recorded chest compres- sions}

The chest compression data recorded by the Resusci Anne SkillReporter and CPRmeter were further analyzed to determine the reasons for the discrepancy in chest compression depth. We found that the reference lines for measuring chest compression depth differed between devices (figure 1).

\section{DISCUSSION}

Accelerometer feedback devices, such as the CPRmeter, were developed to provide chest compression feedback during CPR and have been used in clinical CPR trials. (4-6) Although the visual feedback system of the CPR meter is intuitive and easy to use, the accelerometer measurement system has some weaknesses. Perkins et al. (7) reported that when used on a bed, accelerometer devices overestimate chest compression depth, indicating that these devices report accurate chest compression depths only when used on a hard floor. Oh et al. (8) reported a solution to this problem involving two accelerometers used simultaneously; however, this technique is not available for commercial use.

The results of our study confirmed our hypothesis. The mean chest compression depths measured by the Resusci Anne SkillReporter and CPRmeter were significantly different under complete and incomplete recoil conditions. We determined the reasons for this discrepancy by analyzing the chest compression recorded 


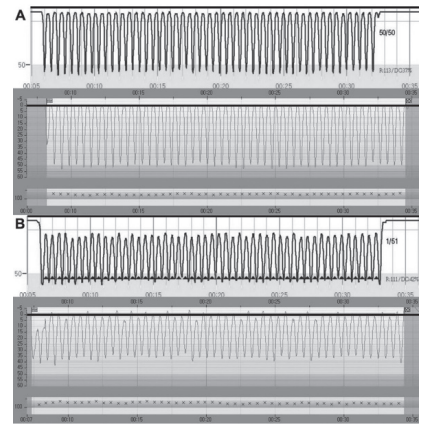

Figure 1. The chest compression data recorded by the Resusci Anne SkillReporter (top) and CPRmeter (below). The Black horizontal lines indicate reference lines for measuring chest compression depths.

A) Experiment 1 (Complete chest wall recoil condition)

B) Experiment 2 (Incomplete chest wall recoil condition).

by the two devices.

The Resusci Anne SkillReporter measures chest compression depths from a fixed reference line based on the chest wall height of the manikin before the rescuer starts chest compressions. Thus, the Resusci Anne SkillReporter accurately measures the depth of the initial chest compression; however, if subsequent compressions do not fully recoil, the manikin overestimates the chest compression depth by the degree of the incomplete recoil depth (figure 2). In contrast, the CPRmeter adjusts the chest compression depth reference (figure 3). Those using the manikin for CPR training or research purposes should be aware of this difference.

Although we confirmed that the chest compression depth measured by the two devices differed, we were unable to determine which measurement was correct. It is possible that the accelerometer overestimated compression depth under the complete recoil condition. If a rescuer's hands are placed above the zero-point, the accelerometer device may "bounce" off the chest wall of the manikin, and thereby estimate a greater than actual depth of compression. Furthermore, we could not confirm the accuracy of the CPRmeter chest
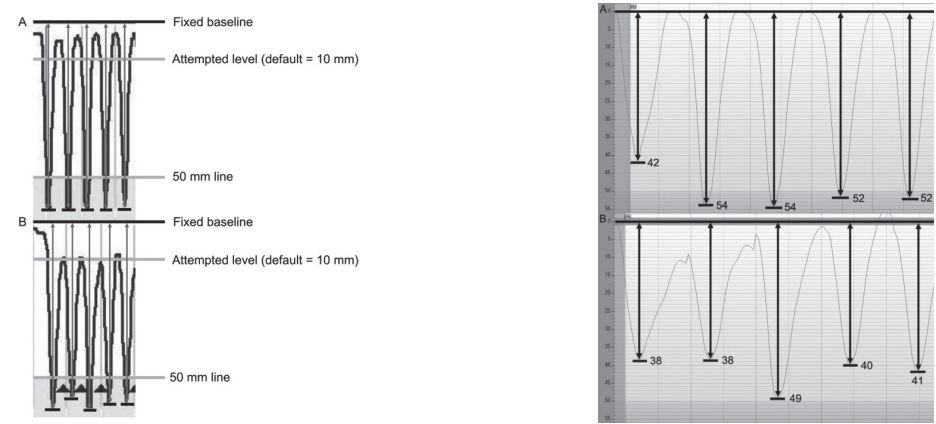

Figure 2. The chest compression data recorded by the Resusci Anne SkillReporter. Grey double-headed arrows indicate measured chest compression depths.

A) Experiment 1 (Complete chest wall recoil condition)

B) Experiment 2 (Incomplete chest wall recoil condition).

compression depth measurements because we did not use the gold standard procedure in our study.

Our findings have several implications. First, incomplete recoil increases the overestimation of compression depth in the Resusci Anne SkillReporter. Second, the Resusci Anne SkillReporter assumes that the chest compression recoils completely when the chest wall is released within $1 \mathrm{~cm}$ of the reference line (indicated in figure 2). Thus, the device may register incomplete recoils as full recoils leading to the overestimation of chest compression depth.

We suggest that using the exact incomplete recoil depth ([average incomplete recoil depth / average actual chest compression depth] ' 100) rather than the incomplete recoil rate ([number of incomplete recoiled chest compressions / total number of chest compressions] ' 100) is more intuitive and readily understood than the method currently used. The actual compression depth (compression depth - incomplete recoil depth) should be recorded simultaneously. This study had several limitations. First, the chest compression depth measurement errors are $\pm 15 \%$ for the Resusci Anne SkillReporter and $\pm 10 \%$ for the CPRmeter according to the manufacturers' specifica-
Figure 3. The chest compression data recorded by the CPRmeter. Black horizontal lines indicate adjusted reference lines for measuring chest compression depths. Black double-headed arrows indicate measured chest compression depths.

A) Experiment 1 (Complete chest wall recoil condition)

B) Experiment 2 (Incomplete chest wall recoil condition).

tions. Because training tools tend to have a higher tolerance for measurement error than those used in research, and as neither device was designed for research purposes, the measurement errors may have contributed to our findings. Second, we evaluated only one type of manikin. Thus, further studies are needed to determine if the same measurement errors would occur using other manikin devices.

\section{CONCLUSIONS}

The chest compression depth measured by the Resusci Anne SkillReporter was significantly different from that of the CPRmeter. Cardiopulmonary resuscitation instructors, trainees, and researchers should be aware of this difference to ensure the most accurate interpretation of their training or experimental results.

\section{FUNDING}

This research was supported by the ChungAng University Research Grants in 2016.

\section{REFERENCES}

1. Finn JC, Bhanji F, Lockey A, Monsieurs K, Frengley R, Iwami T, et al. Part 8: Education, implementation, and teams: 2015 International Consensus on Cardiopulmonary Resuscitation and Emergency Cardiovascular Care Science with Treatment Recommendations. Resuscitation 2015;95:e203-24.

2. Oh JH, Kim CW, Kim SE, Lee SJ, Lee DH. Comparison of chest compressions in the standing position beside a bed at knee level and the kneeling position: a non-randomised, single-blind, cross-over trial. Emerg Med J 2014;31:533-5. 
3. Centre for Clinical Research and Biostatistics. The Chinese University of Hong Kong: Sample size estimation. [Cited 20 January 2015.] Available from URL http://www2.ccrb.cuhk.edu.hk/stat/Means.htm.

4. Buleon C, Parienti JJ, Halbout L, Arrot X, De Facq Regent H, Chelarescu D, et al. Improvement in chest compression quality using a feedback device (CPRmeter): a simulation randomized crossover study. Am J Emerg Med 2013;31:1457-61.

5. Sainio M, Hellevuo H, Huhtala H, Hoppu S, Eilevstjonn J, Tenhunen J, et al. Effect of mattress and bed frame deflection on real chest compression depth measured with two CPR sensors. Resuscitation 2014;85:840-3.

6. Sugerman NT, Edelson DP, Leary M, Weidman EK, Herzberg DL, Vanden Hoek TL, et al. Rescuer fatigue during actual in-hospital cardiopulmonary resuscitation with audiovisual feedback: a prospective multicenter study. Resuscitation 2009;80:981-4.

7. Perkins GD, Kocierz L, Smith SC, McCulloch RA, Davies RP. Compression feedback devices over estimate chest compression depth when performed on a bed. Resuscitation 2009;80:79-82.

8. Oh J, Song Y, Kang B, Kang H, Lim T, Suh Y, et al. The use of dual accelerometers improves measurement of chest compression depth. Resuscitation 2012;83:500-4. 